OPEN ACCESS

Edited by: Zhulong Chan,

Chinese Academy of Sciences, China

Reviewed by:

Vasileios Fotopoulos,

Cyprus University of Technology,

Cyprus

Aurelio Gomez-Cadenas,

Universitat Jaume I, Spain

${ }^{*}$ Correspondence:

Ji-Hong Liu

liujihong@mail.hzau.edu.cn

Specialty section: This article was submitted to Plant Physiology,

a section of the journal

Frontiers in Plant Science

Received: 05 August 2015

Accepted: 22 September 2015

Published: 13 October 2015

Citation:

Liu J-H, Wang W, Wu H, Gong X and Moriguchi T (2015)

Polyamines function in stress tolerance: from synthesis

to regulation.

Front. Plant Sci. 6:827.

doi: 10.3389/fpls.2015.00827

\section{Polyamines function in stress tolerance: from synthesis to regulation}

\author{
Ji-Hong Liu ${ }^{1 *}$, Wei Wang ${ }^{1}$, Hao $W^{1}{ }^{1}$, Xiaoqing Gong ${ }^{1,2}$ and Takaya Moriguchi ${ }^{3}$ \\ ${ }^{1}$ Key Laboratory of Horticultural Plant Biology, College of Horticulture and Forestry Science, Huazhong Agricultural University, \\ Wuhan, China, ${ }^{2}$ State Key Laboratory of Crop Stress Biology for Arid Areas, College of Horticulture, Northwest A\&F \\ University, Yangling, China, ${ }^{3}$ National Institute of Fruit Tree Science, National Agriculture and Food Research Organization, \\ Tsukuba, Japan
}

Plants are challenged by a variety of biotic or abiotic stresses, which can affect their growth and development, productivity, and geographic distribution. In order to survive adverse environmental conditions, plants have evolved various adaptive strategies, among which is the accumulation of metabolites that play protective roles. A wellestablished example of the metabolites that are involved in stress responses, or stress tolerance, is the low-molecular-weight aliphatic polyamines, including putrescine, spermidine, and spermine. The critical role of polyamines in stress tolerance is suggested by several lines of evidence: firstly, the transcript levels of polyamine biosynthetic genes, as well as the activities of the corresponding enzymes, are induced by stresses; secondly, elevation of endogenous polyamine levels by exogenous supply of polyamines, or overexpression of polyamine biosynthetic genes, results in enhanced stress tolerance; and thirdly, a reduction of endogenous polyamines is accompanied by compromised stress tolerance. A number of studies have demonstrated that polyamines function in stress tolerance largely by modulating the homeostasis of reactive oxygen species (ROS) due to their direct, or indirect, roles in regulating antioxidant systems or suppressing ROS production. The transcriptional regulation of polyamine synthesis by transcription factors is also reviewed here. Meanwhile, future perspectives on polyamine research are also suggested.

Keywords: abiotic stress, antioxidant, polyamine, polyamine biosynthesis, ROS, transcriptional regulation

\section{INTRODUCTION}

As sessile organisms, plants are frequently challenged by a variety of adverse biotic or abiotic environmental factors. Since, unlike animals, plants cannot escape from unfavorable environments, harsh stresses constitute major factors that limit growth and development, and severely restrict the production of high-quality agricultural crops. Exposure to the stressful conditions can therefore lead to a substantial difference in potential and actual crop yields, the size of which largely depends on the severity and duration of the environmental stresses in question. Abiotic stresses, such as drought, flooding, extreme temperatures, high salinity, chemical toxicity, nutrient deficiency and others, are regarded as the predominant causes of crop loss and may account for more than $50 \%$ reduction of the yield of the major annual and perennial crops worldwide (Wang et al., 2003). In this regard, understanding how plants adapt to, and survive, the abiotic stresses is important for the efficient exploitation of genetic resources with desirable stress tolerance, and for developing new approaches to enhance stress tolerance. 
Plant evolution has been accompanied by the development of complex and highly coordinated systems that allow adaptation to the stresses, involving signaling cascades that start with signal perception, and result in a variety of stress responses. Recently, significant progresses have been made in elucidating the molecular and genetic pathways involved in stress responses, and a number of key components in the stress signaling cascade events have been identified (Yuan et al., 2013; Wisniewski et al., 2014; Gehan et al., 2015; Shi et al., 2015). In the signal transduction pathway, stress signals are perceived by sensors that are primarily located at the plasma membrane, resulting in the release or activation of various secondary messengers, such as calcium (Ca), ROS (reactive oxygen species), and inositol phosphates, which relay the stress signals and activate downstream components, such as protein kinases and protein phosphatases (Nakashima et al., 2009; Danquah et al., 2014; Liu et al., 2014a; Ma et al., 2015). These proteins orchestrate the balance of protein phosphorylation and play a key role in the regulation of transcription factors (TFs), which bind to cisacting elements in the promotes of their downstream target genes, thereby activating their transcription. This signaling cascade has been shown to be conserved in various plants and allows the plants to survive under the harsh environments (Liu et al., 2014a).

Stress responses are manifested by a range of morphological, physiological, biochemical, and molecular changes. Among these, molecular reprogramming plays a pivotal role, and a large number of studies have described the up- or down-regulation of a wide spectrum of stress-responsive genes (Seki et al., 2002; Thomashow, 2010; Liu et al., 2014a). These genes are generally classified into regulatory or functional types, based on the function of their products. Regulatory genes, encoding protein kinases, phospholipases, and TFs, act as master switches involved in hierarchical signaling cascades, thereby playing vital roles in transcriptional control of downstream stressresponsive genes. The functional genes act directly to mitigate stress-derived injuries via their products, which include a diverse set of metabolites (Shinozaki and Yamaguchi-Shinozaki, 2007). These protective approaches include the stabilization of membranes and macromolecules, alleviation of oxidative stresses, and maintenance of water status. One well studied group of metabolites comprises the polyamines, which have long been suggested to protect and maintain the function and structure of cellular components in response to stresses. Since the first report describing the accumulation of the polyamine putrescine as a result of potassium deficiency (Richards and Coleman, 1952), a large number of studies have implicated polyamines in plant responses to a myriad of abiotic stresses, and these have been reviewed elsewhere (Liu et al., 2007; Kusano et al., 2008; Alcázar et al., 2010a; Hussain et al., 2011; Minocha et al., 2014; Shi and Chan, 2014; Tiburcio et al., 2014). Here, we review recent progress in understanding the association between polyamines and stress responses, with an emphasis on their role in maintenance of ROS homeostasis. In addition, recent advances in identifying and characterizing upstream regulatory genes involved in the stressinduced transcriptional regulation of polyamine metabolism are also discussed.

\section{POLYAMINE SYNTHESIS AND CATABOLISM: CURRENT STATUS}

Polyamines (PAs) are low-molecular-weight, aliphatic polycations that are ubiquitously distributed in all living organisms, including bacteria, animals, and plants (Hussain et al., 2011). There are three major PAs in plants, putrescine (Put), spermidine (Spd), and spermine (Spm), although other types of PAs, such as cadaverine, can also be present. The plant PA biosynthetic pathway has been extensively studied (Kusano et al., 2008; Vera-Sirera et al., 2010; Pegg and Casero, 2011; Gupta et al., 2013) and differs from that of animals in that it involves two precursors, 1 -ornithine and 1 -arginine, to generate putrescine, while only l-ornithine is used in animals. In plants, Put is produced via the catalytic actions of ornithine decarboxylase (ODC, EC 4.1.1.17) and arginine decarboxylase (ADC, EC 4.1.1.19) in three steps. Put is then converted into Spd by Spd synthase (SPDS, EC 2.5.1.16), with the addition of an aminopropyl moiety donated by decarboxylated $S$-adenosylmethionine (dcSAM). dcSAM is synthesized from methionine via two sequential reactions that are catalyzed by methionine adenosyltransferase (EC 2.5.1.6) and S-adenosylmethionine decarboxylase (SAMDC, EC 4.1.1.50), respectively. Spd is then converted into Spm or thermospermine, again using $\mathrm{dcSAM}$ as an aminopropyl donor, in a reaction catalyzed by Spm synthase (SPMS, EC 2.5.1.22) and thermospermine synthase (ACL5, EC 2.5.1.79), respectively (Figure 1). It should be noted that there is no known gene encoding ODC in the sequenced genome of model plant Arabidopsis thaliana (Hanfrey et al., 2001), suggesting that this species may only produce Put via the ADC pathway. Finally, PA synthesis may vary between tissues/organs, one example being that the shoot apical meristem of tobacco (Nicotiana tabacum) serves as the predominant site of Spd and Spm synthesis, while Put is mostly synthesized in roots (Moschou et al., 2008).

Apart from their de novo synthesis, PAs have been shown to undergo catabolism (Figure 1), catalyzed by two classes of enzymes, copper-containing diamine oxidases (CuAOs) and FAD-containing polyamine oxidases (PAOs; Cona et al., 2006). $\mathrm{CuAOs}$ mainly catalyze the oxidation of Put and cadaverine (Cad) at the primary amino groups, producing 4-aminobutanal, peroxide $\left(\mathrm{H}_{2} \mathrm{O}_{2}\right)$ and ammonia (Alcázar et al., 2010a; Moschou et al., 2012). Generally, CuAO proteins exhibit high affinity for Put and Cad than for Spd and Spm (Moschou et al., 2012), although it has been demonstrated that $A$. thaliana $\mathrm{CuAO}$ enzymes can also use Spd as a substrate (Planas-Portell et al., 2013). Plant CuAO proteins are present at high level in dicots, especially in Fabaceous species, such as pea, chickpea, lentil, and soybean seedlings (Cona et al., 2006). Until now, $C u A O$ genes have been identified in only a few plant species, such as A. thaliana (Møller and McPherson, 1998; Planas-Portell et al., 2013) and chickpea (Rea et al., 1998). In $A$. thaliana there are at least 10 putative $\mathrm{CuAO}$ genes, and four of these (AtAO1, AtCuAO1, AtCuAO2, and AtCuAO3) have also been identified (Møller and McPherson, 1998; Planas-Portell et al., 2013).

In contrast to CuAOs, PAOs are present at high levels in monocots and have a high affinity for Spd, Spm, and their derivatives (Alcázar et al., 2010a). Plant PAOs are divided into 


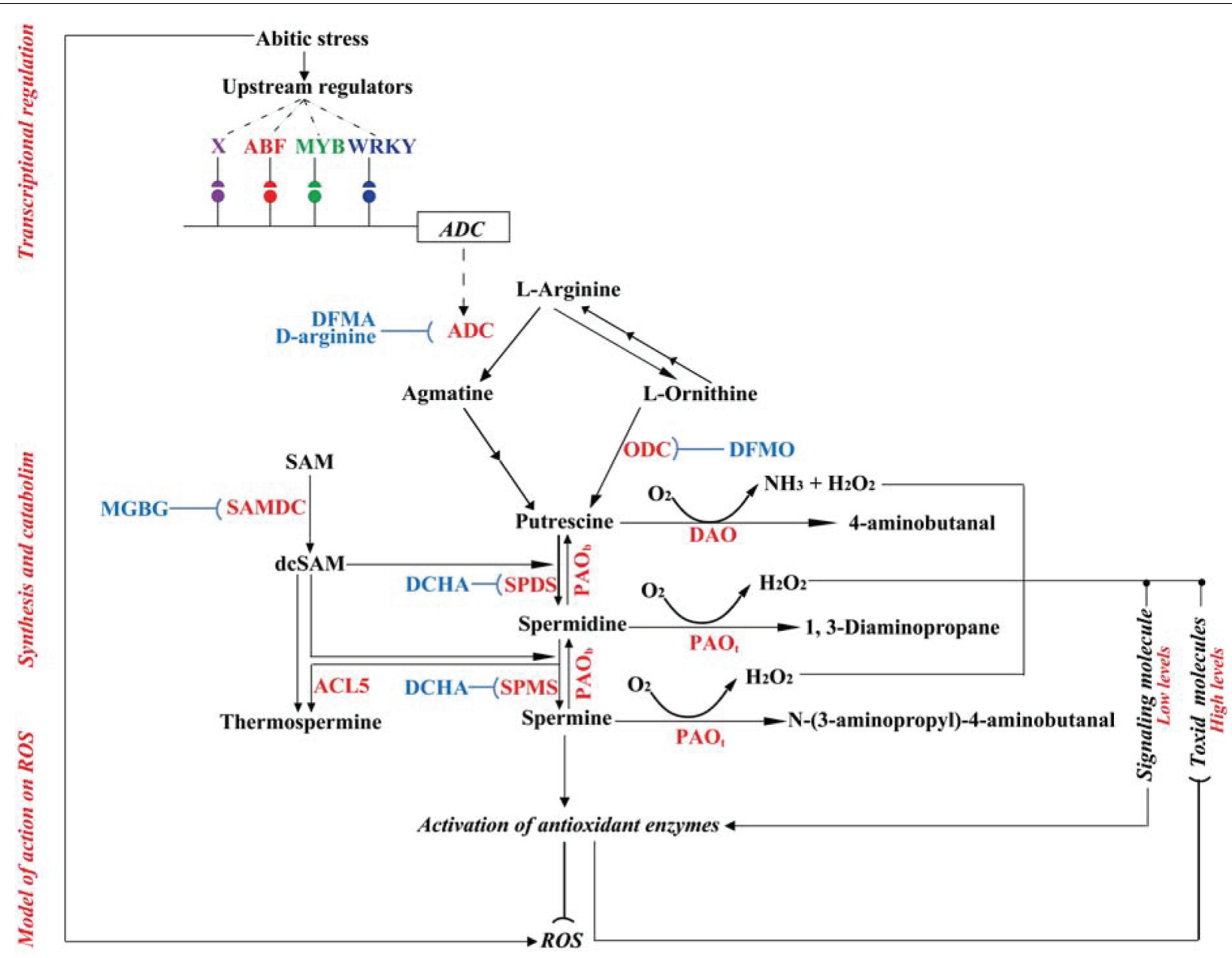

FIGURE 1 | A schematic diagram on synthesis, catabolism, regulation, and action of plant polyamines under abiotic stresses. The enzymes are shown in red, while the inhibitors of the polyamine biosynthetic enzymes are shown in blue. $\mathrm{PAO}_{t}$ indicates the $\mathrm{PAO}$ simplicated in terminal metabolism, whereas $\mathrm{PAO}_{b}$ is involved in back conversion. Circles indicate the relevant cis-acting elements within the ADC promoter. $A D C$ coding sequence is boxed, and the promoter is shown by the horizontal line. $\mathrm{X}$ is an unknown transcription factor that regulates the expression of $A D C$ gene. Arrows mean promotion or stimulation, whereas blunted arrows indicate inhibition, of the related processes. The dashed arrow shows the translation from the ADC gene to ADC protein/enzyme.

two major groups, depending on their potential functions in polyamine catabolism. The first group catalyzes the terminal catabolism of Spd and Spm to produce 1,3-diaminopropane (DAP), $\mathrm{H}_{2} \mathrm{O}_{2}$, and $\mathrm{N}$-(3-aminopropyl)-4-aminobutanal (Spm catabolism), or 4-aminobutanal (Spd catabolism; Cona et al., 2006; Angelini et al., 2010; Moschou et al., 2012). The second group is responsible for PA conversions, in which $\mathrm{Spm}$ is converted back to Spd, and Spd to Put (Moschou et al., 2012; Mo et al., 2015). To date, $P A O$ genes have been identified in several plant species, including $A$. thaliana (Fincato et al., 2011), tobacco (Yoda et al., 2006), rice (Oryza sativa; Ono et al., 2012), barley (Hordeum vulgare; Cervelli et al., 2001), maize (Zea mays; Cervelli et al., 2000), poplar (Tuskan et al., 2006), apple (Malus domestica; Kitashiba et al., 2006), cotton (Gossypium barbadense; Mo et al., 2015), and sweet orange (Citrus sinensis; Wang and Liu, 2015). Plant PAO proteins are encoded by small gene families, as revealed by analyses of fully sequences genomes. There are five, seven, and six $P A O$ genes in A. thaliana, rice, and sweet orange, respectively. However, only few of the $P A O$ genes belonging to the first group have been characterized, and to date only ZmPAO and OsPAO7, from maize and rice, respectively, have been reported to be involved in PA terminal catabolism (Cona et al., 2006; Liu et al., 2014b). In contrast, many PAO genes belonging to the second group have been identified. For example, all five $P A O$ genes from A. thaliana (AtPAO1-AtPAO5) and four PAO genes from rice (OsPAO1, OsPAO3, OsPAO4, and OsPAO5) have been shown to be involved in the back conversion of polyamines (Tavladoraki et al., 2006; Kamada-Nobusada et al., 2008; Moschou et al., 2008; Fincato et al., 2011; Ono et al., 2012; Ahou et al., 2014; Liu et al., 2014b), and recently, a sweet orange PAO gene (CsPAO3) was added to the list of identified genes from this group (Wang and Liu, 2015). As PA catabolism gives rise to the production of $\mathrm{H}_{2} \mathrm{O}_{2}$, which may act either as a signaling molecule at low levels or as a toxic compound when the level is high, the ratio of PA catabolism to biosynthesis has been considered as a crucial factor for induction of tolerance responses or plant cell death under 
abiotic stress (Moschou et al., 2008). This suggests that PA-derived $\mathrm{H}_{2} \mathrm{O}_{2}$ may play a key role in the maintenance of ROS homeostasis, depending on its cellular levels.

\section{INVOLVEMENT OF POLYAMINE METABOLISM IN STRESS RESPONSE}

\section{Change in PA Levels and Transcript Levels of Genes Involved in Polyamine Metabolism Under Abiotic Stress Conditions}

Polyamines have been shown to be involved in various processes associated with plant growth and development, such as embryogenic competence (Silveira et al., 2013), programmed cell death (Kim et al., 2013), fruit ripening (Gil-Amado and GomezJimenez, 2012), xylem differentiation (Tisi et al., 2011), as well as biofilm formation (Lee et al., 2009). Accumulating evidences suggest that plant PAs function in adaptive responses to various environmental stresses, and this is supported by the extensive variation in polyamine levels under stress conditions. Since the first report described Put accumulation under potassium deficiency decades ago (Richards and Coleman, 1952), changes in PA levels have been universally observed in various plant species subjected to a range of abiotic stresses, including drought, high salinity, low and high temperatures, nutrient deficiency, and others (Liu et al., 2007). In some cases, it has been observed that the three most abundant PAs, Put, Spd and Spm, show substantial increases in abundance following abiotic stress (Yang et al., 2007). However, in most cases, only one type of the three PAs shows a significant increase. For example, when apple callus was treated with salt, Put levels increased, while those of Spd and Spm underwent only minor changes (Liu et al., 2006). In contrast, sweet orange callus was reported to show predominant increases in Spd content when exposed to salt and cold stress conditions (Wang and Liu, 2009), and grape (Vitis vinifera) plants showed a dramatic accumulation of Spd and Spm following salt stress (Ikbal et al., 2014). In another study, it was reported that 18 rice varieties exhibited notable changes in Spm levels when grown under long-term drought stress (Do et al., 2014). These findings suggest that PA accumulation is influenced by different factors, such as plant species in question, stress tolerance capacity, stress types and conditions, and the physiological status of the examined tissues/organs. It also indicates the existence of complicated PA dynamics under abiotic stress, which may explain why differing or contradictory results have been reported. The size of PA pool can be correlated with the stress tolerance capacity, further underlining the significance of PAs in providing protection against stresses. Generally, tolerant genotypes accumulate greater amounts of PAs than sensitive genotypes (Hatmi et al., 2015); however, genotypes with contrasting stress tolerance have been shown to display different patterns of PA accumulation under some abiotic stresses. In several studies tolerant genotypes accumulated more Spd and Spm, while the sensitive genotypes from the same plants species accumulated more Put under the same types of stresses (Krishnamurthy and Bhagwat, 1989; Santa-Cruz et al., 1998; Liu et al., 2004). Although whether Spd and Spm play more important roles in counteracting abiotic stress remains to be determined, it is a reasonable hypothesis, since Spd and Spm contain one and two additional primary amino groups $\left(-\mathrm{NH}_{2}\right)$, respectively, compared to Put, allowing them to be more efficient for executing protective functions.

The accumulation of PAs under abiotic stress conditions is largely due to the increased de novo synthesis of free PAs. Since their synthesis is primarily regulated at the transcriptional level, an understanding of the expression patterns of the biosynthetic genes is important for understanding the regulation of PA levels. To this end, a myriad of studies have been carried out to investigate the steady-state transcript levels of PA biosynthetic genes. Available data to date indicate that most of the PA biosynthetic genes, including $A D C, S P D S, S P M S$, and $S A M D C$, are up-regulated by stresses, despite a difference in the timing and degree of induction (Liu et al., 2006, 2008, 2009, 2011; Wang et al., 2011b). Of these genes, $A D C$ is most widely characterized in different plants and has been demonstrated to be a crucial stress-responsive gene (Urano et al., 2004; Liu et al., 2006; Wang et al., 2011b). Increased transcript levels of the PA biosynthetic genes coincide with the accumulation of free PA in some studies, but inconsistent in others (Liu et al., 2006; Wang and Liu, 2009). One reason for the disparity between gene expression profiles and PA accumulation is likely due to PA catabolism. Notably, the expression patterns of PA biosynthetic genes have also been shown to be correlated with stress tolerance (Pillai and Akiyama, 2004). For instance, citrus genotypes with better salt and cold tolerance displayed earlier and/or greater induction of $S A M D C$ transcript at the initial stages of stress treatment (Wang et al., 2010).

\section{Effects of Modulating PA Content on Stress Tolerance}

Polyamine accumulation is usually considered to be a general plant response to abiotic stresses, but the cause-effect relationship between PA accumulation and protection remains unclear. An effective strategy for understanding the roles of PAs in stress tolerance is to modulate their cellular levels, which has been accomplished using three approaches, including their exogenous application, overexpression of their biosynthetic genes and the use of PA synthesis inhibitors.

Exogenous application of Put, Spd, or Spm at different concentrations has been shown to confer enhanced tolerance to various stresses in different plants (Duan et al., 2008). For example, exogenous application of Put considerably enhanced salt tolerance in apple callus and thermotolerance of wheat (Liu et al., 2006; Kumar et al., 2014). A recent study by Zhang et al. (2015b) demonstrated that damage caused by saline-alkaline stress to tomato (Solanum lycopersicum) plants was substantially alleviated when $0.25 \mathrm{mM}$ Spd was applied, and exogenous Spd supplementation can also alleviate salt stress in sorghum (Sorghum bicolor) seedlings (Yin et al., 2015). In another study, Harindra Champa et al. (2015) demonstrated that exogenous application of $1 \mathrm{mM} \mathrm{Spm} \mathrm{reduced} \mathrm{chilling} \mathrm{injury} \mathrm{during} \mathrm{low}$ temperature storage of grape berries, leading to maintenance of fruit quality and shelf life. 
Apart from exogenous PA application, several elegant studies have shown that the overexpression of PA biosynthetic genes is an effective strategy to elevate the endogenous PA pool and to modify stress tolerance. For example, overexpression of $A D C$ genes from oat (Avena sativa) and Datura stramonium resulted in greater accumulation of Put in the transgenic plants, which displayed enhanced drought tolerance when compared with the wild type (WT) genotypes (Roy and Wu, 2001; Capell et al., 2004). Recently, it was shown that constitutive overexpression of $A D C 2$ in $A$. thaliana, oat ADC in Lotus tenuis and PtADC of Poncirus trifoliata in tobacco and tomato noticeably increased drought tolerance in the transgenic plants (Alcázar et al., 2010b; Wang et al., 2011a; Espasandin et al., 2014). In addition, other PA biosynthetic genes, such as $O D C, S A M D C$, and SPDS, have also been overexpressed in transgenic plants, resulting in enhanced tolerance to specific stresses, such as drought and salt (Roy and Wu, 2002; Waie and Rajam, 2003). In summary, overexpression of a PA biosynthetic gene has been demonstrated to confer tolerance to various abiotic stresses (Kasukabe et al., 2004, 2006; Wi et al., 2006; Wang et al., 2011a,b), indicating that changes in the endogenous PA pool has a profound influences on stress tolerance.

Several inhibitors have been identified that repress different PA biosynthetic enzymes, thereby inhibiting endogenous PA synthesis. Their use has provided useful insights into the role of PAs in stress tolerance. Specific or non-specific inhibitors have been used in order to elucidate the role of different PAs. d-arginine, an inhibitor of ADC, was shown to be effective in reducing Put synthesis and its application to apple callus compromised salt tolerance; an effect that was reversed when exogenous Put was applied, suggesting a role for Put in combating salt stress (Liu et al., 2006). Moreover, treatment of grape plants with methylglyoxal-bis(guanylhydrazone) (MGBG), an inhibitor of SAMDC, which is involved in synthesis of Spd and Spm, led to a greater deterioration of plant growth under salt stress than those without MGBG treatment (Ikbal et al., 2014). Recently, it was shown that treatment of sorghum plants with dicyclohexylammonium sulphate (DCHA), an inhibitor of SPDS and SPMS, ameliorated the silicon-induced salt tolerance, implying the positive role of PA in this process (Yin et al., 2015).

\section{ROLE OF POLYAMINES IN STRESS TOLERANCE: FROM AN ANTIOXIDANT PERSPECTIVE}

As mentioned above, elevation of endogenous PA levels is one of the metabolic hallmarks of plants exposed to abiotic stresses (Kusano et al., 2008), implying that they are important for protecting plants against harsh environmental conditions. Nevertheless, in spite of many observations of changes in PA levels under stresses, the precise physiological and molecular mechanisms by which they confer protection remain elusive (Marco et al., 2011). The biological function of the polycationic PAs were initially associated with their capacity to bind anionic macromolecules, such as nucleic acids and proteins, a characteristic that allows PAs to play a role in the regulation of transcription and translation (Bachrach, 2010; Gill and Tuteja,
2010; Igarashi and Kashiwagi, 2010; Tiburcio et al., 2014). They have also been suggested to function in maintaining membrane stability under adverse conditions (Liu et al., 2007; Tiburcio et al., 2014); however, besides other than these mechanisms, there is increasing evidence that their role in stress tolerance is associated with modulating antioxidant systems.

Reactive oxygen species are produced under normal growth conditions, but their homeostasis is a highly coordinated balance between generation and detoxification. Under abiotic stresses, ROS production is elevated, causing excessive ROS accumulation and oxidative stress, which is toxic to living cells due to lipid peroxidation and membrane damage, and can finally result in cell death (Biswas and Mano, 2015). PAs are thought to play a role in modulating ROS homeostasis in two ways. Firstly, they may inhibit the auto-oxidation of metals, which in turn impairs the supply of electrons for the generation of ROS (Shi et al., 2010). They may also directly act as antioxidants and scavenge ROS, although there is no evidence for this mechanism at present. Secondly, PAs may affect antioxidant systems, and a number of studies have demonstrated that priming of plants with polyamines led to increases in endogenous PA contents and concomitant enhanced tolerance to abiotic stresses, such as drought, heat, and cold. The elevation of stress tolerance is concurrent with the activation of antioxidant enzymes. For example, exogenous application of Spm to $P$. trifoliata led to an elevation of POD, SOD, and CAT activities, accompanied by a remarkable decrease in ROS levels under dehydration (Shi et al., 2010). Exogenous supply of Spd to rice seedlings mitigated heat-induced damages, and increased activities of antioxidant enzymes and levels of antioxidant, accompanied by reduced accumulation of $\mathrm{H}_{2} \mathrm{O}_{2}$ (Mostofa et al., 2014). Similar findings have been observed using other plants, such as tobacco, soybean, cucumber, and pistachio (Xu et al., 2011; Radhakrishnan and Lee, 2013; Shu et al., 2013; Kamiab et al., 2014). On the other hand, genetic manipulation of PA biosynthetic genes has been demonstrated to promote stress tolerance via modulation of antioxidant machineries. Overexpression of MdSPDS1 in European pear (Pyrus communis) resulted in an enhanced tolerance to heavy metals, which was largely ascribed to the activation of antioxidant enzymes (Wen et al., 2009). Ectopic expression of PtADC in tobacco and tomato also conferred enhanced dehydration and drought tolerance, coincident with a substantial repression of ROS generation in the transgenic plants (Wang et al., 2011a). Another line of evidence supporting the role of PAs in modulating ROS homeostasis is the use of inhibitors of PA biosynthetic enzymes. As an example, it was shown that the use of D-arginine resulted in a decrease in endogenous PA levels and a consequent increase in ROS accumulation (Wang et al., 2011b; Zhang et al., 2015a). These studies demonstrate that PAs may alleviate the oxidative stress of the stressed plants through regulation of antioxidant systems, along with changes in the ROS production and redox status (Shu et al., 2013; Tanou et al., 2014).

However, a direct link between increased PA levels and antioxidant enzyme activity has yet to be proven. One possibility is that the PAs may function as signaling molecules that can activate the antioxidant enzymes, and indeed Spm has been suggested to act as a signaling molecule (Mitsuya et al., 2009). Another link 
may be the production of $\mathrm{H}_{2} \mathrm{O}_{2}$ by PAO-mediated PA catabolism. An increase in the endogenous PA levels to a certain threshold may promote their degradation, generating $\mathrm{H}_{2} \mathrm{O}_{2}$. It is known that $\mathrm{H}_{2} \mathrm{O}_{2}$ plays dual roles in plant responses to abiotic stresses, one being to act as a regulator of signaling cascades at a low cytosolic concentration, which may contribute to the induction of antioxidant enzymes (Moschou et al., 2008; Zhang et al., 2015a; Figure 1). On the other hand, the PAs may influence various antioxidant enzymes through regulation of their expression. Higher transcript levels of antioxidant enzyme-encoding genes have been detected in tissues treated with exogenous PAs or in the transgenic plants overexpressing PA biosynthetic genes (Tanou et al., 2014; Zhang et al., 2015b).

\section{TRANSCRIPTIONAL REGULATION OF POLYAMINE SYNTHESIS UNDER ABIOTIC STRESS}

Earlier studies demonstrated that the PA biosynthetic genes display disparate expression profiles under abiotic stresses. For example, $P p A D C$ of peach was up-regulated by dehydration, salt, cold, and cadmium (Liu et al., 2009), but repressed by high temperature. It has to be pointed out that the PA biosynthetic genes can be differentially influenced by a particular stress, as exemplified by $M d A D C 1$, which is more responsive to salt stress than other PA biosynthetic genes (Liu et al., 2006). In addition, the transcript levels of PA biosynthetic genes can vary significantly between stress tolerant and stress sensitive genotypes. Such findings suggest that the PA biosynthetic genes may be under tight transcriptional regulation during abiotic stress responses, and so identification and characterization of the associated upstream transcriptional regulators will likely be important in connecting stress responses with PA metabolism.

It has been suggested that $A D C$ acts as an important polyamine biosynthetic gene in response to abiotic stresses, and it has been more extensively characterized than other genes in the pathway. The expression of $A D C$ genes from a number of plant species has been described; in particular those from several species, such as A. thaliana, $P$. trifoliata, have been functionally characterized (Urano et al., 2004; Wang et al., 2011a,b). Thus, ADC genes are promising candidates used for identifying potential transcriptional regulators, such as TFs or protein kinases. The identification and bioinformatics analysis of promoter sequences are common first steps toward identifying potential TFs that regulate a PA biosynthetic gene, prior to the use of yeast onehybrid screening of cDNA libraries. This generally involves characterizing putative cis-acting elements that are present within the promoters. Recently, Basu et al. (2014) reported that in silico analysis of the promoter region of rice SamDC gene revealed the presence of several putative cis-acting elements, such as ABRE, LTRE, MYBR, and W-box, which have been shown to be closely associated with various environmental factors, such as drought, cold, and abscisic acid (ABA) signaling. These findings suggest that the PA biosynthetic genes may be controlled by a common set of TFs, or that a given TF may control different genes involved in PA biosynthesis. This idea is congruent with earlier reports that PA biosynthetic genes, such as PpADC (Liu et al., 2009) or PtADC
(Wang et al., 2011b), are responsive to different stresses. In addition, it also suggests that the endogenous PA levels may be modulated by altering the expression of TFs, either through overexpression or repression (Huang et al., 2010; Chen et al., 2015).

MYB proteins are TFs that play important roles in plant development and stress responses (Dubos et al., 2010). Sun et al. (2014) reported that a stress-responsive R2R3-type MYB gene of P. trifoliata, PtsrMYB, regulated its $A D C$ gene, PtADC. Yeast onehybrid assay demonstrated that $P t s r M Y B$ predominantly interacts with two regions of the PtADC promoter, indicating the PtADC may be a target gene of PtsrMYB. Moreover, overexpression of PtsrMYB led to an increase in mRNA levels of $A D C$ genes in the transgenic lines when compared with WT plants, concurrent with increased PA levels. In a recent study, Chen et al. (2015) showed that overexpression of a cotton MYB TF, GbMYB5, also led to upregulation of three polyamine biosynthetic genes in the transgenic lines. These findings suggest that MYBs might be likely to govern polyamine synthesis under abiotic stresses through regulating the relevant genes.

$\mathrm{ABF}$ is a key $\mathrm{TF}$ involved in the transduction of signals associated with drought and osmotic stress (Yoshida et al., 2010, 2015). It is known that under abiotic stress conditions, synthesis of $\mathrm{ABA}$ is typically increased, which in turn triggers signaling through a network that includes components such as ABA receptors, protein phosphatases, and SnRK proteins. The activated SnRK proteins can in turn phosphorylate ABF TFs, which then regulate downstream target genes (Danquah et al., 2014; Zhang et al., 2015a). In addition, PA biosynthetic genes, such as those encoding ADC, SAMDC, SPDS, have been shown to be induced under drought stress or following ABA treatment, and this is accompanied by increase in the endogenous PAs. However, whether the induction of PA genes or the accumulation of PAs is directly associated with ABA signaling cascades has not yet been addressed. Recently, PtrABF from $P$. trifoliata, an $\mathrm{ABF} 4$ homolog, was shown to regulate the expression of an $A D C$ gene by interacting with the ABRE elements within the $A D C$ promoter. Overexpression of PtrABF greatly increased the mRNA levels of $A D C$, and resulted in an increase in endogenous Put levels, whereas treatment of the PtrABF-overexpressing lines with an ADC inhibitor resulted in a decrease of Put contents, and compromised dehydration tolerance (Huang et al., 2010; Zhang et al., 2015a). These results provide convincing evidence that $A D C$ is a candidate target gene of ABF. Characterization of this regulatory cascade may elucidate the transcriptional regulation of $A D C$ genes and the associated accumulation of PAs under drought or osmotic stresses.

WRKY proteins comprise a large family of TFs in plants and play important roles in regulating the synthesis of several metabolites, such as lignin, phytoalexins, terpenoid indole alkaloid (Suttipanta et al., 2011). Recently, Gong et al. (2015) reported that overexpression of FcWRKY70, a WRKY70 homolog from Fortunella crassifolia, led to the increased expression of $A D C$ genes, whereas suppression of FCWRKY70, using an RNAi approach, down-regulated $A D C$ expression. Put levels were prominently increased in FcWRKY70-overexpressing lines, but decreased in the RNAi lines when compared to the WT. 
In addition, FcWRKY70 can interact with the W-box elements in the $A D C$ promoter. Taken together, these results indicate that FcWRKY70 may also act as a positive regulator of $A D C$ expression and regulate Put synthesis under abiotic stresses.

The above data suggests that $A D C$ genes may be under the control of members from different TF families, including ABF, WRKY, and MYB proteins (Figure 1). However, TFs from other families may also be involved in the regulation of $A D C$ gene expression (Huang et al., 2015). Of note, all of the TFs mentioned above are proposed as positive regulators of PA biosynthesis and $A D C$ expression, while TFs that may negatively regulate PA gene expression have not been characterized. In addition, it is not known whether other PA biosynthetic genes, such as those encoding SAMDC, SPDS and SPMS proteins, are regulated by the same TFs mentioned above.

Apart from the TFs, other regulatory proteins may also control PA biosynthetic gene expression and PA accumulation. One of these is mitogen-activated protein kinase (MAPK), which has been shown to play roles in various signaling pathways related to plant development and stress responses. Huang et al. (2011) reported that the expression of NtADC1 and NtSAMDC, two tobacco genes involved in PA biosynthesis, was induced in transgenic tobacco plants overexpressing PtrMAPK of trifoliate orange. It can be inferred from this result that MAPK may phosphorylate the two corresponding proteins, but this needs to be verified in the future.

\section{PERSPECTIVES AND CONCLUDING REMARKS}

Polyamines are considered to play important roles in protecting plant cells from stress-associated damages. To date, tremendous progresses have been made in understanding the significance of PAs in stress responses. There is accumulating evidence that PA levels undergo extensive changes in response to a range of abiotic stresses, and physiological, molecular and genetic approaches have been used to identify and functionally characterize PA biosynthetic genes in various plant species. These efforts underpin our understanding of the role of PAs in counteracting adverse environmental cues, and provide valuable information for enhancing stress tolerance through the modulation of cellular PA levels via exogenous PA application,

\section{REFERENCES}

Ahou, A., Martignago, D., Alabdallah, O., Tavazza, R., Stano, P., Macone, A., et al. (2014). A plant spermine oxidase/dehydrogenase regulated by the proteasome and polyamines. J. Exp. Bot. 65, 1585-1603. doi: 10.1093/jxb/eru016

Alcázar, R., Altabella, T., Marco, F., Bortolotti, C., Reymond, M., Koncz, C., et al. (2010a). Polyamines: molecules with regulatory functions in plant abiotic stress tolerance. Planta 231, 1237-1249. doi: 10.1007/s00425-0101130-0

Alcázar, R., Planas, J., Saxena, T., Zarza, X., Bortolotti, C., Cuevas, J., et al. (2010b). Putrescine accumulation confers drought tolerance in transgenic Arabidopsis plants over-expressing the homologous Arginine decarboxylase 2 gene. Plant Physiol. Bioch. 48, 547-552. doi: 10.1016/j.plaphy.2010.02.002

Angelini, R., Cona, A., Federico, R., Fincato, P., Tavladoraki, P., and Tisi, A. (2010). Plant amine oxidases "on the move": an update. Plant Physiol. Bioch. 48, 560-564. doi: 10.1016/j.plaphy.2010.02.001 or the transgenic manipulation of PA biosynthetic genes. Nevertheless, many key questions remain unanswered. First, the causal relationship between PA accumulation and stress tolerance has not been determined, despite numerous observations of changes in PA levels in response to abiotic stresses. Second, the cellular compartmentation and transportation of PAs is not well understood, although a few PA transporters have been identified (Fujita et al., 2012; Mulangi et al., 2012). In addition, the mode of action of PAs in enhancing stress tolerance has not been definitely established, although several possible models have been proposed. One example is the scarcity of direct evidence confirming the involvement of PAs in the activation of antioxidant enzymes for ROS detoxification. Last, the signaling cascades linking stress responses and PA genes are still far from being well defined. To date, TFs regulating $A D C$ genes have been identified, but those that regulate other PA biosynthetic genes are unknown. In keeping with these unanswered questions, there are several promising areas of future study. First, the sites of PA production and actions in plant cells need to be identified and to this end, the cellular localization of PAs and their transporters should be determined. Second, the physiological and molecular mechanisms concerning the roles of PAs in stress tolerance need to be elucidated, and in particular, how PAs contribute to the activation of antioxidant enzymes and ROS removal should be clearly deciphered. Last but not the least, the molecular mechanisms underlying the accumulation of PAs in response to abiotic stresses, including the PA biosynthetic genes and the transcriptional regulation network associated with those genes, must be defined. This information will advance our understanding of PA accumulation and gene expression, and can be incorporated with physiological, biochemical, molecular and genetic approaches to better understand the complex regulation of PA synthesis under abiotic stresses, as well as the cross talk between different TF-mediated signaling pathways.

\section{ACKNOWLEDGMENTS}

This work was financially supported by the National Science Foundation of China $(31272147,31572100)$, the Ministry of Agriculture, and the National High Technology Research and Development Program of China (2011AA100205).

Bachrach, U. (2010). The early history of polyamine research. Plant Physiol. Bioch. 48, 490-495. doi: 10.1016/j.plaphy.2010.02.003

Basu, S., Roychoudhury, A., and Sengupta, D. N. (2014). Identification of transactingfactors regulating SamDC expression in Oryza sativa. Biochem. Biophys. Res. Commun. 445, 398-403. doi: 10.1016/j.bbrc.2014. 02.004

Biswas, M. S., and Mano, J. (2015). Lipid peroxide-derived short-chain carbonyls mediate $\mathrm{H}_{2} \mathrm{O}_{2}$-induced and $\mathrm{NaCl}$-induced programmed cell death in plants. Plant Physiol. 168, 885-898. doi: 10.1104/pp.115.256834

Capell, T., Bassie, L., and Christou, P. (2004). Modulation of the polyamine biosynthetic pathway in transgenic rice confers tolerance to drought stress. Proc Natl. Acad. Sci. U.S.A. 101, 9909-9914. doi: 10.1073/pnas.0306974101

Cervelli, M., Cona, A., Angelini, R., Polticelli, F., Federico, R., and Mariottini, P. (2001). A barley polyamine oxidase isoform with distinct structural features and subcellular localization. Eur. J. Biochem. 268, 3816-3830. doi: 10.1046/j.14321327.2001.02296.x 
Cervelli, M., Tavladoraki, P., Agostino, S. D., Angelini, R., Federico, R., and Mariottini, P. (2000). Isolation and characterization of three polyamine oxidase genes from Zea mays. Plant Physiol. Bioch. 38, 667-677. doi: 10.1016/S09819428(00)01170-0

Chen, T., Li, W., Hu, X., Guo, J., Liu, A., and Zhang, B. L. (2015). A cotton MYB transcription factor, GbMYB5, is positively involved in plant adaptive response to drought stress. Plant Cell Physiol. 56, 917-929. doi: 10.1093/pcp/pcv019

Cona, A., Rea, G., Angelini, R., Federico, R., and Tavladoraki, P. (2006). Function of amine oxidases in plant development and defence. Trends Plant Sci. 11, 80-88. doi: 10.1016/j.tplants.2005.12.009

Danquah, A., de Zelicout, A., Colcombet, J., and Hirt, H. (2014). The role of ABA and MAPK signaling pathways in plant abiotic stress responses. Biotechnol. Adv. 32, 40-52. doi: 10.1016/j.biotechadv.2013.09.006

Do, P. T., Drechsel, O., Heyer, A. G., Hincha, D. K., and Zuther, E. (2014). Changes in free polyamine levels, expression of polyamine biosynthesis genes, and performance of rice cultivars under salt stress: a comparison with responses to drought. Front. Plant Sci. 5:182. doi: 10.3389/fpls.2014.00182

Duan, J., Li, J., Guo, S., and Kang, Y. (2008). Exogenous spermidine affects polyamine metabolism in salinity-stressed Cucumis sativus roots and enhances short-term salinity tolerance. J. Plant Physiol. 165, 1620-1635. doi: 10.1016/j.jplph.2007.11.006

Dubos, C., Stracke, R., Grotewold, E., Weisshaar, B., Martin, C., and Lepiniec, L. (2010). MYB transcription factors in Arabidopsis. Trends Plant Sci. 15, 573-581. doi: 10.1016/j.tplants.2010.06.005

Espasandin, F. D., Maiale, S. J., Calzadilla, P., Ruiz, O. A., and Sansberro, P. A. (2014). Transcriptional regulation of 9-cis-epoxycarotenoid dioxygenase (NCED) gene by putrescine accumulation positively modulates ABA synthesis and drought tolerance in Lotus tenuis plants. Plant Physiol. Bioch. 76, 29-35. doi: 10.1016/j.plaphy.2013.12.018

Fincato, P., Moschou, P. N., Spedaletti, V., Tavazza, R., Angelini, R., Federico, R., et al. (2011). Functional diversity inside the Arabidopsis polyamine oxidase gene family. J. Exp. Bot. 62, 1155-1168. doi: 10.1093/jxb/erq341

Fujita, M., Fujita, Y., Iuchi, S., Yamada, K., Kobayashi, Y., Urano, K., et al. (2012). Natural variation in a polyamine transporter determines paraquat tolerance in Arabidopsis. Proc. Natl. Acad. Sci. U.S.A. 109, 6343-6347. doi: 10.1073/pnas.1121406109

Gehan, M. A., Greenham, K., Mockler, T. C., and McClung, C. R. (2015). Transcriptional networks-crops, clocks, and abiotic stress. Curr. Opin. Plant Biol. 24, 39-46. doi: 10.1016/j.pbi.2015.01.004

Gil-Amado, J. A., and Gomez-Jimenez, M. C. (2012). Regulation of polyamine metabolism and biosynthetic gene expression during olive mature-fruit abscission. Planta 235, 1221-1237. doi: 10.1007/s00425-011-1570-1

Gill, S. S., and Tuteja, N. (2010). Polyamines and abiotic stress tolerance in plants. Plant Signal. Behav. 5, 26-33. doi: 10.4161/psb.5.1.10291

Gong, X. Q., Zhang, J. Y., Hu, J. B., Wang, W., Wu, H., Zhang, Q. H., et al. (2015). FcWRKY70, a WRKY protein of Fortunella crassifolia, functions in drought tolerance and modulates putrescine synthesis by regulating arginine decarboxylase gene. Plant Cell Environ. doi: 10.1111/pce.12539

Gupta, K., Dey, A., and Gupta, B. (2013). Plant polyamines in abiotic stress responses. Acta Physiol. Plant. 35, 2015-2036. doi: 10.1007/s11738-013-1239-4

Hanfrey, C., Sommer, S., Mayer, M. J., Burtin, D., and Michael, A. J. (2001). Arabidopsis polyamine biosynthesis: absence of ornithine decarboxylase and the mechanism of arginine decarboxylase activity. Plant J. 27, 551-560. doi: 10.1046/j.1365-313X.2001.01100.x

Harindra Champa, W. A., Gilla, M. I. S., Mahajanb, B. V. C., and Bedic, S. (2015). Exogenous treatment of spermine to maintain quality and extend postharvest life of table grapes (Vitis vinifera L.) cv. Flame Seedless under low temperature storage. LWT Food Sci Technol. 60, 412-419. doi: 10.1016/j.lwt.2014.08.044

Hatmi, S., Gruau, C., Trotel-Aziz, P., Villaume, S., Rabenoelina, F., Baillieul, F., et al. (2015). Drought stress tolerance in grapevine involves activation of polyamine oxidation contributing to improved immune response and low susceptibility to Botrytis cinerea. J. Exp. Bot. 66, 775-787. doi: 10.1093/jxb/eru436

Huang, X. S., Huo, T., Fu, X. Z., Fan, Q. J., and Liu, J. H. (2011). Cloning, molecular characterization of a mitogen-activated protein kinase gene from Poncirus trifoliata and its ectopic expression confers dehydration/drought tolerance in transgenic tobacco. J. Exp. Bot. 62, 5191-5206. doi: 10.1093/jxb/err229

Huang, X. S., Liu, J. H., and Chen, X. J. (2010). Overexpression of PtrABF gene, a bZIP transcription factor isolated from Poncirus trifoliata, enhances dehydration and drought tolerance in tobacco via scavenging ROS and modulating expression of stress-responsive genes. BMC Plant Biol. 10:230. doi: 10.1186/1471-2229-10-230

Huang, X. S., Zhang, Q. H., Zhu, D. X., Fu, X. Z., Wang, M., Zhang, Q., et al. (2015). ICE1 of Poncirus trifoliata functions in cold tolerance by modulating polyamine levels through interacting with arginine decarboxylase. J. Exp. Bot. 66, 3259-3274. doi: 10.1093/jxb/erv138

Hussain, S. S., Ali, M., Ahmad, M., and Siddique, K. H. (2011). Polyamines: natural and engineered abiotic and biotic stress tolerance in plants. Biotechnol. Adv. 29, 300-311. doi: 10.1016/j.biotechadv.2011.01.003

Igarashi, K., and Kashiwagi, K. (2010). Modulation of cellular function by polyamines. Intl. J. Biochem. Cell. Biol. 42, 39-51. doi: 10.1016/j.biocel.2009.07.009

Ikbal, F. E., Hernández, J. A., Barba-Espín, G., Koussa, T., Aziz, A., Faize, M., et al. (2014). Enhanced salt-induced antioxidative responses involve a contribution of polyamine biosynthesis in grapevine plants. J. Plant Physiol. 171, 779-788. doi: 10.1016/j.jplph.2014.02.006

Kamada-Nobusada, T., Hayashi, M., Fukazawa, M., Sakakibara, H., and Nishimura, M. (2008). A putative peroxisomal polyamine oxidase, AtPAO4, is involved in polyamine catabolism in Arabidopsis thaliana. Plant Cell Physiol. 49, 1272-1282. doi: $10.1093 / \mathrm{pcp} / \mathrm{pcn} 114$

Kamiab, F., Talaie, A., Khezri, S., and Javanshah, A. (2014). Exogenous application of free polyamines enhance salt tolerance of pistachio (Pistacia vera L.) seedlings. Plant Growth Regul. 72, 257-268. doi: 10.1007/s10725-013-9857-9

Kasukabe, Y., He, L., Nada, K., Misawa, S., Ihara, I., and Tachibana, S. (2004). Overexpression of spermidine synthase enhances tolerance to multiple environmental stresses and up-regulates the expression of various stressregulated genes in transgenic Arabidopsis thaliana. Plant Cell Physiol. 45, 712-722. doi: 10.1093/pcp/pch083

Kasukabe, Y., He, L., Watakabe, Y., Otani, M., Shimada, T., and Tachibana, S. (2006). Improvement of environmental stress tolerance of sweet potato by introduction of genes for spermidine synthase. Plant Biotechnol. 23, 75-83. doi: 10.5511/plantbiotechnology.23.75

Kim, N. H., Kim, B. S., and Hwang, B. K. (2013). Pepper arginine decarboxylase is required for polyamine and gamma-aminobutyric acid signaling in cell death and defense response. Plant Physiol. 162, 2067-2083. doi: 10.1104/pp.113.217372

Kitashiba, H., Honda, C., and Moriguchi, T. (2006). Identification of polyamine oxidase genes from apple and expression analysis during fruit development and cell growth. Plant Biotechnol. 23, 425-429. doi: 10.5511/plantbiotechnology.23.425

Krishnamurthy, R., and Bhagwat, K. A. (1989). Polyamines as modulators of salt tolerance in rice cultivars. Plant Physiol. 91, 500-504. doi: 10.1104/pp.91.2.500

Kumar, R. R., Sharma, S. K., Rai, G. K., Singh, K., Choudhury, M., Dhawan, G., et al. (2014). Exogenous application of putrescine at pre-anthesis enhances the thermotolerance of wheat (Triticum aestivum L.). Indian J. Biochem. Biophys. 51, 396-406.

Kusano, T., Berberich, T., Tateda, C., and Takahashi, Y. (2008). Polyamines: essential factors for growth and survival. Planta 228, 367-381. doi: 10.1007/s00425-0080772-7

Lee, J., Sperandio, V., Frantz, D. E., Longgood, J., Camilli, A., Phillips, M. A., et al. (2009). An alternative polyamine biosynthetic pathway is widespread in bacteria and essential for biofilm formation in Vibrio cholerae. J. Biol. Chem. 284, 9899-9907. doi: 10.1074/jbc.M900110200

Liu, H. P., Dong, B. H., Zhang, Y. Y., Liu, Z. P., and Liu, Y. L. (2004). Relationship between osmotic stress and the levels of free, conjugated, and bound polyamines in leaves of wheat seedlings. Plant Sci. 166, 1261-1267. doi: 10.1016/j.plantsci.2003.12.039

Liu, J. H., Ban, Y., Wen, X. P., Nakajima, I., and Moriguchi, T. (2009). Molecular cloning and expression analysis of an arginine decarboxylase gene from peach (Prunus persica). Gene 429, 10-17. doi: 10.1016/j.gene.2008.10.003

Liu, J. H., Inoue, H., and Moriguchi, T. (2008). Salt stress-mediated changes in free polyamine titers and expression of genes responsible for polyamine biosynthesis of apple in vitro shoots. Environ. Exp. Bot. 62, 28-35. doi: 10.1016/j.envexpbot.2007.07.002

Liu, J. H., Kitashiba, H., Wang, J., Ban, Y., and Moriguchi, T. (2007). Polyamines and their ability to provide environmental stress tolerance to plants. Plant Biotechnol. 24, 117-126. doi: 10.5511/plantbiotechnology.24.117

Liu, J. H., Nada, K., Honda, C., Kitashiba, H., Wen, X. P., Pang, X. M., et al. (2006). Polyamine biosynthesis of apple callus under salt stress: importance of the 
arginine decarboxylase pathway in stress response. J. Exp. Bot. 57, 2589-2599. doi: $10.1093 / \mathrm{jxb} / \mathrm{erl} 018$

Liu, J. H., Nakajima, I., and Moriguchi, T. (2011). Effects of salt and osmotic stresses on free polyamine content and expression of polyamine biosynthetic genes in Vitis vinifera. Biol. Plantarum 55, 340-344. doi: 10.1007/s10535-011-0050-6

Liu, J. H., Peng, T., and Dai, W. S. (2014a). Critical cis-acting elements and interacting transcription factors: key players associated with abiotic stress responses in plants. Plant Mol. Biol. Rep. 32, 303-317. doi: 10.1007/s11105-0130667-z

Liu, T., Kim, D. W., Niitsu, M., Maeda, S., Watanabe, M., Kamio, Y., et al. (2014b). Polyamine oxidase 7 is a terminal catabolism-type enzyme in Oryza sativa and is specifically expressed in anthers. Plant Cell Physiol. 55, 1110-1122. doi: $10.1093 / \mathrm{pcp} / \mathrm{pcu} 047$

Ma, Y., Dai, X., Xu, Y., Luo, W., Zheng, X., Zeng, D., et al. (2015). COLD1 confers chilling tolerance in rice. Cell 160, 1209-1221, doi: 10.1016/j.cell.2015.01.046

Marco, F., Alcazar, R., Tiburcio, A. F., and Carrasco, P. (2011). Interactions between polyamines and abiotic stress pathway responses unraveled by transcriptome analysis of polyamine overproducers. OMICS $15,775-781$. doi: 10.1089/omi.2011.0084

Minocha, R., Majumdar, R., and Minocha, S. C. (2014). Polyamines and abiotic stress in plants: a complex relationship. Front. Plant Sci. 5:175. doi: $10.3389 /$ fpls.2014.00175

Mitsuya, Y., Takahashi, Y., Berberich, T., Miyazaki, A., Matsumura, H., Takahashi, H., et al. (2009). Spermine signaling plays a significant role in the defense response of Arabidopsis thaliana to cucumber mosaic virus. J. Plant Physiol. 166, 626-643. doi: 10.1016/j.jplph.2008.08.006

Møller, S. G., and McPherson, M. J. (1998). Developmental expression and biochemical analysis of the Arabidopsis ataol gene encoding an $\mathrm{H}_{2} \mathrm{O}_{2}$ generating diamine oxidase. Plant J. 13, 781-791. doi: 10.1046/j.1365313X.1998.00080.x

Mo, H., Wang, X., Zhang, Y., Zhang, G., Zhang, J. F., and Ma, Z. Y. (2015). Cotton polyamine oxidase is required for spermine and camalexin signalling in the defence response to Verticillium dahliae. Plant J. 83, 962-975. doi: 10.1111/tpj.12941

Moschou, P. N., Paschalidis, K. A., Delis, I. D., Andriopoulou, A. H., Lagiotis, G. D., Yakoumakis, D. I., et al. (2008). Spermidine exodus and oxidation in the apoplast induced by abiotic stress is responsible for $\mathrm{H}_{2} \mathrm{O}_{2}$ signatures that direct tolerance responses in tobacco. Plant Cell 20, 1708-1724. doi: 10.1105/tpc.108.059733

Moschou, P. N., Wu, J., Cona, A., Tavladoraki, P., Angelini, R., and RoubelakisAngelakis, K. A. (2012). The polyamines and their catabolic products are significant players in the turnover of nitrogenous molecules in plants. J. Exp. Bot. 63, 5003-5015. doi: 10.1093/jxb/ers202

Mostofa, M. G., Yoshida, N., and Fujita, M. (2014). Spermidine pretreatment enhances heat tolerance in rice seedlings through modulating antioxidative and glyoxalase systems. Plant Growth Regul. 73, 31-44. doi: 10.1007/s10725-0139865-9

Mulangi, V., Phuntumart, V., Aouida, M., Ramotar, D., and Morris, P. (2012). Functional analysis of OsPUT1, a rice polyamine uptake transporter. Planta 235, 1-11. doi: 10.1007/s00425-011-1486-9

Nakashima, K., Ito, Y., and Yamaguchi-Shinozaki, K. (2009). Transcriptional regulatory networks in response to abiotic stresses in Arabidopsis and grasses. Plant Physiol. 149, 88-95. doi: 10.1104/pp.108.129791

Ono, Y., Kim, D. W., Watanabe, K., Sasaki, A., Niitsu,M., Berberich, T., et al. (2012). Constitutively and highly expressed Oryza sativa polyamine oxidases localize in peroxisomes and catalyze polyamine back conversion. Amino Acids 42, 867-876. doi: $10.1007 / \mathrm{s} 00726-011-1002-3$

Pegg, A. E., and Casero, R. A. Jr. (2011). Current status of the polyamine research field. Methods Mol. Biol. 720, 3-35. doi: 10.1007/978-1-61779-034-8_1

Pillai, M. A., and Akiyama, T. (2004). Differential expression of an S-adenosyl-Lmethionine decarboxylase gene involved in polyamine biosynthesis under low temperature stress in japonica and indica rice genotypes. Mol. Genet. Genomics 271, 141-149. doi: 10.1007/s00438-003-0963-7

Planas-Portell, J., Gallart, M., Tiburcio, A. F., and Altabella, T. (2013). Coppercontaining amine oxidases contribute to terminal polyamine oxidation in peroxisomes and apoplast of Arabidopsis thaliana. BMC Plant Biol. 13:109. doi: 10.1186/1471-2229-13-109

Radhakrishnan, R., and Lee, I. J. (2013). Spermine promotes acclimation to osmotic stress by modifying antioxidant, abscisic acid, and jasmonic acid signals in soybean. J. Plant Growth Regul. 32, 22-30. doi: 10.1007/s00344-012-9274-8
Rea, G., Laurenzi, M., Tranquilli, E., D’Ovidio, R., Federico, R., and Angelini, R. (1998). Developmentally and wound-regulated expression of the gene encoding a cell wall copper amine oxidase in chickpea seedlings. FEBS Lett. 437, 177-182. doi: 10.1016/S0014-5793(98)01219-8

Richards, F. J., and Coleman, R. G. (1952). Occurrence of putrescine in potassium deficient barley. Nature 170, 479-481. doi: 10.1038/17 0460a0

Roy, M., and Wu, R. (2001). Arginine decarboxylase transgene expression and analysis of environmental stress tolerance in transgenic rice. Plant Sci. 160, 869-875. doi: 10.1016/S0168-9452(01)00337-5

Roy, M., and Wu, R. (2002). Overexpression of S-adenosylmethionine decarboxylase gene in rice increases polyamine level and enhances sodium chloride-stress tolerance. Plant Sci. 163, 987-992. doi: 10.1016/S0168-9452(02)00272-8

Santa-Cruz, A., Perez-Alfocea, F., Caro, M., and Acosta, M. (1998). Polyamines as short-term salt tolerance traits in tomato. Plant Sci. 138, 9-16. doi: 10.1016/S0168-9452(98)00143-5

Seki, M., Narusaka, M., Ishida, J., Nanjo, T., Fujita, M., Oono, Y., et al. (2002). Monitoring the expression profiles of ca. 7000 Arabidopsis genes under drought, cold, and high-salinity stresses using a full-length cDNA microarray. Plant J. 31, 279-292. doi: 10.1046/j.1365-313X.2002.01359.x

Shi, H., and Chan, Z. (2014). Improvement of plant abiotic stress tolerance through modulation of the polyamine pathway. J. Intg. Plant Biol. 56, 114-121. doi: $10.1111 /$ jipb. 12128

Shi, J., Fu, X. Z., Peng, T., Huang, X. S., Fan, Q. J., and Liu, J. H. (2010). Spermine pretreatment confers dehydration tolerance of citrus in vitro plants via modulation of antioxidative capacity and stomatal response. Tree Physiol. 30, 914-922. doi: 10.1093/treephys/tpq030

Shinozaki, K., and Yamaguchi-Shinozaki, K. (2007). Gene networks involved in drought stress response and tolerance. J. Exp. Bot. 58, 221-227. doi: 10.1093/jxb/erl164

Shi, Y., Ding, Y., and Yang, S. (2015). Cold signal transduction and its interplay with phytohormones during cold acclimation. Plant Cell Physiol. 56, 7-15. doi: $10.1093 /$ pcp/pcu115

Shu, S., Yuan, L. Y., Guo, S. R., Sun, J., and Yuan, Y. H. (2013). Effects of exogenous spermine on chlorophyll fluorescence, antioxidant system and ultrastructure of chloroplasts in Cucumis sativus L. under salt stress. Plant Physiol. Bioch. 63, 209-216. doi: 10.1016/j.plaphy.2012.11.028

Silveira, V., De Vita, A. M., Macedo, A. F., Dias, M. F. R., Floh, E. I. S., and Santa- Catarina, C. (2013). Morphological and polyamine content changes in embryogenic and non-embryogenic callus of sugarcane. Plant Cell Tiss. Organ Cult. 114, 351-364. doi: 10.1007/s11240-013-0330-2

Suttipanta, N., Pattanaik, S., Kulshrestha, M., Patra, B., Singh, S. K., and Yuan, L. (2011). The transcription factor CrWRKY1 positively regulates the terpenoid indole alkaloid biosynthesis in Catharanthus roseus. Plant Physiol. 157, 2081-2093. doi: 10.1104/pp.111.181834

Sun, P. P., Zhu, X. F., Huang, X. S., and Liu, J. H. (2014). Overexpression of a stressresponsive MYB transcription factor of Poncirus trifoliata confers enhanced dehydration tolerance and increases polyamine biosynthesis. Plant Physiol. Bioch. 78, 71-79. doi: 10.1016/j.plaphy.2014.02.022

Tanou, G., Ziogas, V., Belghazi, M., Christou, A., Filippou, P., Job, D., et al. (2014). Polyamines reprogram oxidative and nitrosative status and the proteome of citrus plants exposed to salinity stress. Plant Cell Environ. 37, 864-885. doi: 10.1111/pce.12204

Tavladoraki, P., Rossi, M. N., Saccuti, G., Perez-Amador, M. A., Polticelli, F., Angelini, R., et al. (2006). Heterologous expression and biochemical characterization of a polyamine oxidase from Arabidopsis involved in polyamine back conversion. Plant Physiol. 141, 1519-1532. doi: 10.1104/pp.106. 080911

Thomashow, M. F. (2010). Molecular basis of plant cold acclimation: insights gained from studying the CBF cold response pathway. Plant Physiol. 154, 571-577. doi: 10.1104/pp.110.161794

Tiburcio, A. F., Altabella, T., Bitrián, M., and Alcázar, R. (2014). The roles of polyamines during the lifespan of plants: from development to stress. Planta 240 , 1-18. doi: $10.1007 / \mathrm{s} 00425-014-2055-9$

Tisi, A., Federico, R., Moreno, S., Lucretti, S., Moschou, P. N., Roubelakis-Angelakis, K. A., et al. (2011). Perturbation of polyamine catabolism can strongly affect root development and xylem differentiation. Plant Physiol. 157, 200-215. doi: 10.1104/pp.111.173153 
Tuskan, G. A., DiFazio, S., Jansson, S., Bohlmann, J., Grigoriev, I., Hellsten, U., et al. (2006). The genome of black cottonwood, Populus trichocarpa (Torr. \& Gray). Science 313, 1596-1604. doi: 10.1126/science. 1128691

Urano, K., Yoshiba, Y., Nanjo, T., Ito, T., Yamaguchi-Shinozaki, K., and Shinozaki, K. (2004). Arabidopsis stress-inducible gene for arginine decarboxylase AtADC2 is required for accumulation of putrescine in salt tolerance. Biochem. Biophys. Res. Commun. 313, 369-375. doi: 10.1016/j.bbrc.2003. 11.119

Vera-Sirera, F., Minguet, E. G., Singh, S. K., Ljung, K., Tuominen, H., Blazquez, M. A., et al. (2010). Role of polyamines in plant vascular development. Plant Physiol. Bioch. 48, 534-539. doi: 10.1016/j.plaphy.2010.01.011

Waie, B., and Rajam, M. V. (2003). Effect of increased polyamine biosynthesis on stress responses in transgenic tobacco by introduction of human $S$ adenosylmethionine gene. Plant Sci. 164, 727-734. doi: 10.1016/S01689452(03)00030-X

Wang, B. Q., Zhang, Q. F., Liu, J. H., and Li, G. H. (2011a). Overexpression of PtADC confers enhanced dehydration and drought tolerance in transgenic tobacco and tomato: effect on ROS elimination. Biochem. Biophys. Res. Commun. 413, 10-16. doi: 10.1016/j.bbrc.2011.08.015

Wang, J., Sun, P. P., Chen, C. L., Wang, Y., Fu, X. Z., and Liu, J. H. (2011b). An arginine decarboxylase gene PtADC from Poncirus trifoliata confers abiotic stress tolerance and promotes primary root growth in Arabidopsis. J. Exp. Bot. 62, 2899-2914. doi: 10.1093/jxb/erq463

Wang, J., Liu, J. H., Yu, A., Xiang, Y., Kurosawa, T., Nada, K., et al. (2010). Cloning, biochemical identification, and expression analysis of a gene encoding $S$-adenosylmethionine decarboxylase in Citrus sinensis Osbeck. J. Hortic. Sci. Biotechnol. 85, 219-226.

Wang, J., and Liu, J. H. (2009). Change in free polyamine contents and expression profiles of two polyamine biosynthetic genes in citrus embryogenic callus under abiotic stresses. Biotechnol. Biotechnol. Eq. 29, 1289-1293. doi: 10.1080/13102818.2009.10817655

Wang, W., and Liu, J. H. (2015). Genome-wide identification and expression analysis of the polyamine oxidase gene family in sweet orange (Citrus sinensis). Gene 555, 421-429. doi: 10.1016/j.gene.2014. 11.042

Wang, W., Vinocur, B., and Altman, A. (2003). Plant responses to drought, salinity and extreme temperatures: towards genetic engineering for stress tolerance. Planta 218, 1-14. doi: 10.1007/s00425-003-1105-5

Wen, X. P., Ban, Y., Inoue, H., Matsuda, N., and Moriguchi, T. (2009). Spermidine levels are implicated in heavy metal tolerance in a spermidine synthase overexpressing transgenic European pear by exerting antioxidant activities. Transgenic Res. 19, 91-103. doi: 10.1007/s11248-0099296-6

Wi, S. J., Kim, W. T., and Park, K. Y. (2006). Overexpression of carnation Sadenosylmethionine decarboxylase gene generates a broad spectrum tolerance to abiotic stresses in transgenic tobacco plants. Plant Cell Rep. 25, 1111-1121. doi: 10.1007/s00299-006-0160-3
Wisniewski, M., Nassuth, N., Teulières, C., Marque, C., Rowland, J., Cao, P. B., et al. (2014). Genomics of cold hardiness in woody plants. Crit. Rev. Plant Sci. 33, 92-124. doi: 10.1080/07352689.2014.870408

Xu, S., Hu, J., Li, Y., Ma, W., Zheng, Y., and Zhu, S. J. (2011). Chilling tolerance in Nicotiana tabacum induced by seed priming with putrescine. Plant Growth Regul. 63, 279-290. doi: 10.1007/s10725-010-9528-Z

Yang, J. C., Zhang, J. H., Liu, K., Wang, Z. Q., and Liu, L. J. (2007). Involvement of polyamines in the drought resistance of rice. J. Exp. Bot. 58, 1545-1555. doi: $10.1093 / \mathrm{jxb} / \mathrm{erm} 032$

Yin, L., Wang, S., Tanaka, K., Fujihara, S., Itai, A., Den, X., et al. (2015). Siliconmediated changes in polyamines participate in silicon-induced salt tolerance in Sorghum bicolor L. Plant Cell Environ. doi: 10.1111/pce.12521 [Epub ahead of print].

Yoda, H., Hiroi, Y., and Sano, H. (2006). Polyamine oxidase is one of the key elements for oxidative burst to induce programmed cell death in tobacco cultured cells. Plant Physiol. 142, 193-206. doi: 10.1104/pp.106.080515

Yoshida, T., Fujita, Y., Maruyama, K., Mogami, J., Todaka, D., Shinozaki, K., et al. (2015). Four Arabidopsis AREB/ABF transcription factors function predominantly in gene expression downstream of SnRK2 kinases in abscisic acid signalling in response to osmotic stress. Plant Cell Environ. 38, 35-49. doi: $10.1111 /$ pce. 12351

Yoshida T., Fujita, Y., Sayama, H., Kidokoro, S., Maruyama, K., Mizoi, J., et al. (2010). AREB1, AREB2, and ABF3 are master transcription factors that cooperatively regulate ABRE-dependent ABA signaling involved in drought stress tolerance and require ABA for full activation. Plant J. 61, 672-685. doi: 10.1111/j.1365-313X.2009.04092.x

Yuan, L., Liu, X., Luo, M., Yang, S., and Wu, K. (2013). Involvement of histone modifications in plant abiotic stress responses. J. Intg. Plant Biol. 55, 892-901. doi: $10.1111 /$ jipb.12060

Zhang, Q. H., Wang, M., Hu, J. B., Wang, W., Fu, X. Z., and Liu, J. H. (2015a). PtrABF of Poncirus trifoliata functions in dehydration tolerance by reducing stomatal density and maintaining reactive oxygen species homeostasis. J. Exp. Bot. 66, 5911-5927. doi: 10.1093/jxb/erv301

Zhang, Y., Zhang, H., Zou, Z. R., Liu, Y., and Hu, X. H. (2015b). Deciphering the protective role of spermidine against saline-alkaline stress at physiological and proteomic levels in tomato. Phytochemistry 110, 13-21. doi: $10.1016 /$ j.phytochem.2014.12.021

Conflict of Interest Statement: The authors declare that the research was conducted in the absence of any commercial or financial relationships that could be construed as a potential conflict of interest.

Copyright (C) 2015 Liu, Wang, Wu, Gong and Moriguchi. This is an open-access article distributed under the terms of the Creative Commons Attribution License (CC BY).

The use, distribution or reproduction in other forums is permitted, provided the original author(s) or licensor are credited and that the original publication in this journal is cited, in accordance with accepted academic practice. No use, distribution or reproduction is permitted which does not comply with these terms. 